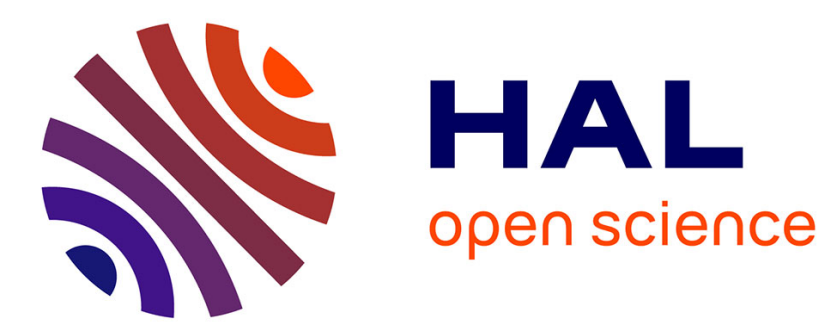

\title{
THE PAIR POTENTIAL APPROACH FOR INTERFACES : FUNDAMENTAL PROBLEMS AND PRACTICAL SOLUTIONS
}

\author{
A. Maggs, N. Ashcroft
}

\section{- To cite this version:}

A. Maggs, N. Ashcroft. THE PAIR POTENTIAL APPROACH FOR INTERFACES : FUNDAMENTAL PROBLEMS AND PRACTICAL SOLUTIONS. Journal de Physique Colloques, 1988, 49 (C5), pp.C5-131-C5-138. 10.1051/jphyscol:1988510 • jpa-00228007

\section{HAL Id: jpa-00228007 https://hal.science/jpa-00228007}

Submitted on 1 Jan 1988

HAL is a multi-disciplinary open access archive for the deposit and dissemination of scientific research documents, whether they are published or not. The documents may come from teaching and research institutions in France or abroad, or from public or private research centers.
L'archive ouverte pluridisciplinaire HAL, est destinée au dépôt et à la diffusion de documents scientifiques de niveau recherche, publiés ou non, émanant des établissements d'enseignement et de recherche français ou étrangers, des laboratoires publics ou privés. 
THE PAIR POTENTIAL APPROACH FOR INTERFACES : FUNDAMENTAL PROBLEMS AND PRACTICAL SOLUTIONS ${ }^{(1)}$

\author{
A.C. MAGGS and N.W. ASHCROFT \\ Laboratory of Atomic and Solid state Physics, Clark Hall, Cornell \\ University, Ithaca, NY 14853-2501, U.S.A.
}

\begin{abstract}
A fundamental problem in the use of a central pair-force model for defect problems is that it omits three-body and higher terms which are necessarily present in real systems. Electronic fluctuation effects are also usually omitted. While these can be small in the simple metals, they are significant in noble and transition metals, as shown by a simple real space argument. To gauge the importance of their effects in interface problems, the structure of a simple $\sum 5$ twist boundary is examined, with the atoms described by both pair- and three-center interactions and as a function of the relative strength of the two.
\end{abstract}

\title{
I. INTRODUCTION
}

In this article we address the applicability of an almost universal assumption made in discussing the energetics of grain boundaries: this is the assumed existence of pairwise central forces between atoms. We do not discuss here the methods with which such calculations are carried out and the resulting structures themselves. These matters are fully described in the review articles by Sutton and Vitek ${ }^{1}$ and elsewhere.

The development that follows will be concerned with an elemental system; this is for purposes of convenience only, and it is clear that the ideas will be applicable mutatis mutandis to alloy systems. Suppose we have an assembly of nuclei and electrons, the nuclei having coordinates $\left\{\vec{R}_{1}, \ldots, \vec{R}_{N}\right\}$ with $N \sim 10^{23}$ in a practical case. Because the nuclei are overwhelmingly massive compared with the electrons, the statics and dynamics of the system can for most situations be described by the Hamiltonian

$$
H\left(\vec{R}_{1}, . ., \vec{R}_{N}\right)=\sum_{i=1}^{N} \frac{P_{i}^{2}}{2 M}+\Phi\left(\vec{R}_{1}, . ., \vec{R}_{N} ; V\right)
$$

where $\vec{P}_{i}$ is the momentum of the nucleus $i$ (whose mass is $M$ ) and $\Phi\left(\vec{R}_{1}, . ., \vec{R}_{N} ; V\right)$ is the total $N$-particle potential energy when the nuclei are in the configuration $\vec{R}_{1}, \ldots, \vec{R}_{N}$. The volume V is included as a variable of $\Phi$ because in general the states of $H$ are not necessarily insulating (among other things the state of a system might depend on external boundary conditions such as the pressure). If the system is metallic or possesses wide election bands, as in a semiconductor, then in the solution of the electronic problem ${ }^{2}$ (whose trace leads to (1)), there arise large volume dependent and structure independent terms. These are exceedingly important in metallic systems, which will form the focus of this paper.

(1) Work supported by DOE Contract No DEFG02-85ER45211 


\section{PAIR POTENTIALS}

Before we specify the special arrangements of atoms (i.e., the selection of the $\vec{R}_{i}$ ) that are pertinent to the interface problem, we need first to enquire about the functional forms expected on general grounds for $\Phi$. This question is of fundamental significance in treating bulk systems, bounded systems, and systems with low symmetry, especially those which lack the simplifying features of a space group. In systems with disorder or with important defects it is physically and certainly conceptually desirable to attempt to express the $N$-particle quantity $\Phi$ as a sum of effective pairwise potentials $\phi_{i j}$ (again, they may be functions of volume). This replacement is justifiable from first principles arguments based on linear screening for simple metals describable by pseudopotentials ${ }^{3}$ and also from fluctuating multipole expansions which in lowest order lead to the familiar Van der Waals potentials. ${ }^{4}$ Most solids of technological interest lack these simplifying characterizations. Nevertheless, it is commonplace to assert that $\Phi\left(R_{1}, . ., R_{N} ; V\right)$ can be written as

$$
\Phi\left(R_{1}, . ., R_{N} ; V\right)=\frac{1}{2} \sum_{i, j}^{\prime} \phi\left(r_{i j}\right)
$$

or

$$
\Phi\left(R_{1}, . ., R_{N} ; V\right)=\Phi_{\circ}(V)+\frac{1}{2} \sum_{i, j}^{\prime} \phi\left(r_{i j}, V\right)
$$

While (3) is more soundly based in metals than (2), neither is completely satisfactory in describing the stability of interfaces. Extended defects are generally analyzed ${ }^{5}$ by omitting three- and highercenter interactions, which by quite general arguments are expected from the systematic expansion of $\Phi\left(R_{1}, \ldots, R_{N} ; V\right)$. Since short range repulsion is dominant, the consequent constraints from geometric packing would seem to make this an a priori reasonable starting point. Nevertheless more distant regions of potentials do play an important role.

For molecular crystals it is easy to extract a dimensionless parameter that gauges the importance of three-center relative to two-center potentials. An ion at the origin experiences an electronic fluctuation leading to an instantaneous dipole $\hat{d}$ (the magnitude of the fluctuation is governed by the polarizability of the ion). The field of this dipole, at a second ion a distance $r$ away is $\hat{d} / r^{3}$, and this induces a dipole of strength $\alpha \hat{d} / r^{3}$. The inducing and induced dipoles lead to the mutual lowering of energy by an amount proportional to $-\alpha^{2} \frac{\langle d d\rangle}{r^{3}}$. This familiar argument is readily generalized by adding a third ion at distance $\vec{r}^{\prime}$ from the origin. The induced dipole $\alpha \hat{d} / r^{3}$ in the second ion then induces a dipole of strength $\left(\alpha \hat{d} / r^{3}\right)_{\left[\vec{r}-\vec{r} T^{3}\right.}$ in the third, which interacts with the first to give an explicitly three body energy proportional to $\alpha^{3}<\hat{d} \hat{d} \hat{d}>\left|r^{3} r^{3}\right| \vec{r}-\left.\vec{r}^{\prime}\right|^{3}$ (the Axilrod-Teller form). If we take a triangular arrangement as an example, then apart form minor combinatorial factors, the ratio of three-body to two-body is given by $\alpha \rho^{(1)}$ where $\rho^{(1)} \sim 1 / r^{3}$ is the average atomic density. Since $\rho^{(1)} \sim 10^{23} / \mathrm{cm}^{3}$, it is clear that if $\alpha$ is as large as a few $(A)^{3}$, then three-body terms arising from fluctuating multipole terms in quasilocalized charge are expected to be important. It is obvious that these kinds of interactions depend on angular displacements as well as on separations. In this respect they differ crucially from the pair-potentials. The condition $\alpha \rho^{(1)} \lesssim_{1}$ is satisfied for molecular systems in the heavier elements. An example is xenon; many-body forces have been shown to be important in its cohesive properties ${ }^{6}$ and also in its critical behavior near the gas-liquid transition. ${ }^{7}$

However, this Van der Waals behavior is also present in the metallic state, both intrinsically in the uniform electron gas ${ }^{7}$ and also in quasilocalized charge such as the electronic distribution characterized by the $d$-orbitals in the noble metals. It is important to recognize that fluctuating multipole terms are direct manifestations of electron-electron correlation effects; as such they are fundamental but are included only in approximate form by self-consistent band theoretic approaches which at every step proceed from the construction of a one-electron (mean field) potential. Thus is a metal like copper, silver, or gold, with complete $d$-shells, the contribution to 
the cohesive energy from the $d$-electrons can be developed from a viewpoint which is quite different from the band structure technique and in some respects is preferable since the real space method involved leads quite naturally to bonds. As shown by Moriarty, ${ }^{8}$ band theory in combination with local density functional theory can lead to a systematic development of the energy of the system in terms of transferable multicenter interactions. This had earlier been noted in the somewhat more restrictive context of tight-binding by Carlsson and Ashcroft. ${ }^{9}$ However, the importance of going beyond pair terms has been emphasized by Finnis, et al. ${ }^{10}$

Whether or not the terminology "Van der Waals metal" is appropriate in a given situation depends entirely on the relative importance of electron correlation effects originating in the quasilocalized charge. The criterion can be framed in terms of the magnitude of the polarizability of the bound charge, which in turn is gauged by the polarizability $\alpha$ of the free ions. As noted above, when this is sufficiently large that the parameter $\alpha \rho$ is significant, then multicenter interactions are expected to be important. However, in a metal there are also pair interactions originating from the monopole terms so that for fluctuation effects to be relevant in the first place it is also necessary that they are significant in terms of the statically screened ion-ion interactions. More precisely, it is necessary that they be at least comparable to the differences in structural energies produced by such terms. As shown recently by Maggs and Ashcroft, ${ }^{11}$ this situation prevails in the noble metals and may also prevail in some of the transition metals. Three body forces, inherently dependent on angular separations, are therefore important in these systems.

\section{THREE- AND HIGHER-ATOM POTENTIALS}

The standard argument given above for establishing the $r^{-6}$ form of the fluctuating dipole dipole (Van der Waals) interaction is readily generalized to the case where the dipoles are embedded in an electron gas whose plasma frequency is $\omega_{p}$. The point is that if the dipole is endowed with a frequency $\omega$, then the corresponding field is now dynamically screened by the frequency dependent dielectric constant of the electron gas. If the fluctuations are dominated by a single excitation energy $\Delta$ (as is the standard Lorentz Oscillator model), then the frequency dependent polarizability is $\alpha(\omega)=\alpha(o) \Delta^{2} /\left(\Delta^{2}-\omega^{2}\right)$ and we find an approximate formula for the screened Van der Waals interaction of a metal with polarizable ions, namely

$$
\Phi_{p}(r)=-\frac{3 \Delta}{4} \frac{\alpha^{2}(0)}{r^{6}}\left(\frac{\Delta}{\Delta+\omega_{p}}\right)^{3}
$$

which reduces to the standard result for an insulator when $\omega_{p} \rightarrow 0$. This result can be compared immediately with the simplest screened point ion result for static interactions

$$
\phi_{i}(r)=\frac{Z^{2} e^{2}}{r}\left(\exp -k_{T F} r\right)
$$

(where $k_{T F}$ is the Thomas-Fermi screening length) or perhaps more appropriately with the standard linear screening pseudopotential result

$$
\phi_{p s}(r)=\frac{1}{(2 \pi)^{3}} \int \overrightarrow{d q} e^{-i \vec{q} \cdot \vec{r}} \frac{4 \pi Z^{2} e^{2}}{q^{2}}\left\{1+\left(\frac{V_{p s}(q)}{V_{c}(q)}\right)^{2}\left(\frac{1}{\epsilon(q)}-1\right)\right\}
$$

where $V_{p s}(q)$ is the electron ion pseudopotential, and $V_{c}(q)$ is the electron-ion interaction were the ion to be a true point ion. In $(6) \epsilon(q)$ is the density dependent static dielectric constant of the fully interacting electron gas. The results of calculations using (6) and its generalizations to non-local pseudopotentials lead to pair interactions that have a principal minimum which is typically a few milli-Rydbergs (a scale which is immediately expected from (5) since $k_{T F} \sim 1 / a_{o}$, and characteristic near neighbor separations are several $a_{o}$ ). From (4) we see that comparable 
energies can be expected from fluctuating dipole interactions if $\alpha$ is one to two $\AA^{3}$ or so. The key point here is that while molecular interactions are indeed small compared with bare Coulomb interactions, they are not small compared with screened Coulomb interactions, and for structural purposes it is to the latter that comparison should properly be made.

Given this observation, we can immediately ask what forms the possible three-particle equivalents of (4) might take, that is, three ion interactions that depend specifically on the polarizability $\alpha$. There is, first of all, a screened version of the Axilrod-Teller interaction described earlier for the insulating case; thus

$$
\phi^{(3)}\left(\vec{r}, \vec{r}^{\prime}\right)=\frac{9}{16} \frac{\alpha^{3}(0) \Delta}{r^{3} r^{13} r^{\prime 3}}\left(\frac{\Delta}{\Delta+\omega_{p}}\right)^{5} f
$$

where $r^{\prime \prime}=\left|\vec{r}^{\prime}-\vec{r}\right|$ and $f=1+3\left(\hat{r}^{\cdot} \hat{r}^{\prime}\right)\left(\hat{r}^{\prime} \cdot \vec{r}^{\prime \prime}\right)\left(\hat{r}^{\prime \prime} \cdot \hat{r}\right)$ is numerically of order 1 . By the argument given earlier if $\alpha \rho^{(1)}$ is appreciable, (which is also the condition that (4) is comparable to (5)-or (6)) to then the three-particle terms (7) will be comparable to the pair interactions. However, in the presence of conduction electrons ( 7$)$ is not the only three atom term. There are also terms in involving $\alpha^{2}(o), \alpha(o)$ and even a three atom term lacking $\alpha$ altogether and originating entirely with the free electrons. These incorporate higher order response functions of the electron gas, ${ }^{6}$ but apart from this their functional forms are similar to (7). It might be expected that such terms would automatically be smaller since in the perturbation sense they are associated with higher orders in the electron-electron interaction. This is not the case; the point is that nonfluctuating terms involve the static response, and this is constrained by the perfect screening sum rule $\left(\frac{\lim }{q \rightarrow 0} \epsilon^{-1}(q, 0)=0\right)$; in contrast the fluctuating terms, which require $\epsilon(q, \omega)$, satisfy no such constraint, and the corresponding interactions $4 \pi e^{2} / q^{2} \epsilon(q, \omega)$ are not small. It is important to note that the three-ion terms are not derivable from any band theoretic approach in which the corresponding corrections are not already included in the exchange-correlation functional. The fact that the three particle interactions as predicted by the method of multipole expansions are apparently larger than those obtained from mean field approaches ${ }^{8}$ certainly deserves further scrutiny. Moreover, it is clear that the validity of the multipole method is limited by the fact that in many practical systems the extent of the "localized charge" is larger than is physically reasonable for the expansion to be completely satisfactory. On the other hand, it is important to recognize that there exists no fundamental physical requirement that three particle forces shall necessarily be smaller than two-particle at long range. This appears to be a possibility when $\alpha$ is sufficiently large (but still within physically reasonable bounds).

\section{ATOM-ATOM INTERACTIONS AND INTERFACE STRUCTURE}

In order to test the possible significance of three-ion interactions on grain boundary structure and stability and to practical solutions to the pair potential problem, we have studied a simple case, namely, a $\sum 5$ twist boundary at the confluence of two (100) faces of FCC crystals. To clearly separate out the effects, we have simplified the sum of (4) and (5) so that jointly they are represented by an Van der Waals 12-6 potential, except that the defining constants are allowed to be dependent on local density. Thus

$$
\phi^{(2)}(r, V)=4 \epsilon\left((\sigma / r)^{12}-(\sigma / r)^{6}\right)
$$

for the pair term. All of the forms for the triplet potential that incorporate the ionic polarizability have the structure

$$
\phi^{(3)}\left(\vec{r}, \vec{r}^{\prime}, \vec{r}^{\prime \prime}\right)=C \frac{1}{r^{3} r^{\prime 3} r^{\prime 3}} f\left(\vec{r}, \vec{r}^{\prime}, \vec{r}^{\prime \prime}\right)
$$

where $f=1+3\left(\hat{r} \cdot \hat{r}^{\prime}\right)\left(\hat{r}^{\prime} \cdot \hat{r}^{\prime \prime}\right)\left(\hat{r}^{\prime \prime} \cdot \hat{r}\right)$. Note that $\phi^{(3)}$ will be cut off at short range by core-core exchange repulsion. The problem is now well posed; we simply relax the atoms of our structure according the (8) and (9). With $\mathrm{C}$ set zero the interface is described by the standard pair 
approximation. Increasing $\mathrm{C}$ from zero introduces triplet interactions into the problem. Notice that it is even possible in this scheme to examine the stability of triplet interactions alone (with appropriate short range repulsion appended).

Under the action of pair forces alone we verify what is well known, namely, that the simulated positions taken up by the atoms in the boundary are largely determined by simple geometrical packing constraints originating with the hard core repulsive region of the pair potential. The largest relaxation occurs exactly at the boundary itself. $\mathrm{I} t$ is important to note that this conclusion is dependent on the simple form of the pair potential used (see below).

If we now add three-body interactions, so that the total potential energy of interaction is a sum of terms of the form

$$
\phi / 4 \epsilon=\left[(\sigma / r)^{12}-(\sigma / r)^{6}\right]=A\left(1+3\left(\hat{r}^{\prime} \hat{r}^{\prime}\right)\left(\hat{r}^{\prime} \cdot \hat{r}^{\prime \prime}\right)\left(\hat{r}^{\prime \prime} \cdot \hat{r}\right)\right) / r^{3} r^{\prime 3} r^{\prime \prime 3}
$$

the simulation can be repeated efficiently by starting with the positions of the ions stabilized under the action of the pair terms. The maximum value of $A$ is taken to be 0.01 ; to set this in context, the "pair bond" energy of $(10)$ is typically $\sim-0.25$, and in relative terms the "triangle" energy for equal bond lengths is 0.29 (i.e., quite large). For this value of $A$ we find the additional relaxation of the atoms to be only about 10 percent of pair relaxations, but interestingly enough, they are two to three times larger in a direction perpendicular to the interface than they are parallel to it. Perhaps more importantly, the effects of the three-body terms are largest in the second plane from the interface and not in the interface itself. It should be noted that Bristowe and Crocker ${ }^{12}$ achieve a similar result but with the use of pair forces alone.

In their study of relaxation at a grain boundary, Budai et $\mathbf{l}^{5}$ were not able to determine a unique pair potential which, when simulated, could yield displacements at the boundary even half as large as those observed experimentally. Though the results described above show that large three-body terms can have observable effects, ${ }^{13}$ these are not comparable to changes that can result from even a minor alteration in the short range repulsive pairwise interaction. But this is precisely where density dependence can play a significant role in systems where the net pair force results from dynamically screened fluctuating-dipole effects and statically screened pseudopotential contributions. As shown by Mon et al, ${ }^{14}$ these two contributions have different density dependences; moreover, if the core-polarizability is sufficiently large, the pair potential at short range can acquire non-monotonic behavior (i.e., there are two relatively close positions where a pair would achieve zero force). Large displacements at a grain boundary are consistent with such a form of microscopic interaction, though as noted by $\mathrm{Oh}$ and Vitek ${ }^{15}$ other interpretations originating in structural multiplicity are also possible.

\section{REFERENCES AND FOOTNOTES}

1. A. P. Sutton and V. Vitek, Phil. Trans. Roy. Soc., A $\underline{309}$ 1, 37, 55 (1983)

2. For a derivation of (1) starting from the two component view of the constitution of matter (nuclei and electrons) see N. W. Ashcroft, "The Liquid State of Matter: Fluids Simple and Complex" E. W. Montroll and J. L. Lebowitz (Eds.) North Holland (1982), p. 141.

3. See, for example, N. W. Ashcroft in "Interatomic Potentials and the Simulation of Lattice Defects" edited by P. C. Gehlen, J. R. Beeler, and R. I. Jaffee (Plenum, New York, 1972) p. 91.

4. A. Dalgarno and W. D. Davidson, Adv. At. Mol. Phys. 2, (1966).

5. J. Budai, P. D. Bristowe, and S. L. Sass, Acta. Metall, Vol. 31, No. 5 pp. 699-712 (1983).

6. A. C. Maggs and N. W. Ashcroft, Phys. Rev. B xx, xxxx (1987) to appear. 
7. R. Goldstein, A. Parola, N. W. Ashcroft, M. W. Pestak, M. H. W. Chan, J. R. deBruyn, and D. A. Balzarini, Phys. Rev. Letts. $\underline{58}, 41$ (1987).

8. J. A. Moriarty, Phys. Rev. Letts. $\underline{55}, 1502$ (1985); see also J. A. Moriarty, Phys. Rev B $\underline{34}$, 6738 (1986).

9. A. E. Carlsson and N. W. Ashcroft, Phys. Rev. B 27, 2101 (1983).

10. M. W. Finnis, K. L. Kear, and D. G. Pettifor, Phys. Rev. Letts. $\underline{52}, 291$ (1984); see also D. G. Pettifor, J. Phys. F $\underline{8}, 219$ (1978).

11. A. C. Maggs and N. W. Ashcroft, Phys. Rev. Letts. 58,113 (1987).

12. P. D. Bristowe and A. G. Crocker, Phil. Mag. A 38487 (1978).

13. For a more detailed account see A. C. Maggs and N. W. Ashcroft (in preparation).

14. K. K. Mon, N. W. Ashcroft, and G. V. Chester Phys. Rev. B $\underline{19}, 5103$ (1979).

15. Y. Oh and V. Vitek, Acta. Metall 34, 1941 (1986).

M.I. Baskes: Would you comment on the uniqueness of pair potentials?

N.W. Ashcroft: I don't know of any rigorous proof that the decomposition of the $\mathrm{N}$-particle potential $\Phi\left(\mathrm{R}_{1}, \ldots, \mathrm{R}_{\mathrm{N}}\right.$; V) into a volume term, pair term, triplet term, ... and so forth, is necessarily unique. But if one proceeds from perturbation theory after tracing out the electron degrees of freedom, the proposition seems reasonable. on the other hand, if one starts from approximations to the $N$-particle problem, then it is not clear a unique potential would result. To give an example from the theory of liquids, the equations of state obtained from the virial route and compressibility route are different. If these were to be "inverted" (a gedanken exercise) to obtain the underlying pair potential, the results would clearly differ. We have to keep in mind that we are trying to deal with a many-body problem.

W. Iojkowski: Could you comment, in the view of your talk, on the physical background of the tight bonding approximation?

N.W. Ashcroft: This question is partly addressed in our recent paper (Maggs and Ashcroft, Phys. Rev. Letters, July 13, 1987). As shown by Moriarity, Finnis, Pettifor, Carlsson and others a systematic development of pair and multi center interactions can be achieved by starting with a set of d-bands. Now, these techniques will obviously apply to bands that result from a tight-binding approach. The difference here is that we are including effects that go beyond the 